\title{
Risk in anthropic environments: methodologies for risk evaluation and exposition reduction
}

\author{
F. Russo \& A. Vitetta \\ Department of Computer Science, Mathematics, \\ Electronics and Transportation, University of Reggio Calabria, Italy
}

\begin{abstract}
The simulation of an anthropic environments system in emergency conditions requires the study of two strictly connected models: risk analysis and evacuation design. To reduce the exposure component of the risk, evacuation measures have to be implemented. When an event happens in a system or is forecasted in the short term, the evacuation measures have to be applied and in some cases have to be designed in real time. This paper is developed with the following main objectives: the formalization of the risk problem; the definition of the probability (or the frequency) that the event occurs, the vulnerability and the exposition with particular reference to a transportation system; the specification of evacuation measures for the reduction of exposition and risk.
\end{abstract}

Keywords: risk analysis, evacuation, simulation, network design.

\section{Introduction}

This paper proposes methods for the simulation and design of a transportation system under conditions of safety and/or security. Safety and security problems are connected with events that generate emergency conditions, such as the 9/11 attack, Atocha station, and the Asian tsunami. Methods for planning a transportation system in an urban area when exogenous events affecting the system occur and/or in emergency conditions, have received little attention from transportation and risk researchers as well as research institutions and journals. Emergency conditions related to safety and/or security problems in a transportation system can be activated by exogenous events (power failure, radiation leak, etc.) or by endogenous events to the transportation system (dangerous emissions, etc.). The dangers for the population could be immediate (such as the emission or the discharge of hazardous goods from a truck, 
earthquake, bomb attack, etc.) or delayed (such as flooding, tsunami, lava flow, power station failure, hurricane, toxic or radioactive cloud, tsunami, etc.).

The developments of decision support systems for emergency conditions have not received much attention in the literature. Only specific aspects are treated concerning large-scale emergencies when a nuclear event occurs [1], in urban systems when general hazards occur $[2,3]$ and in buildings during fires $[4,5]$.

In general, there is no systematic analysis of the general risk theory applied in the transportation system and very often the vulnerability and exposure in the transportation system are considered as similar variables, or in other cases the exposure variables are treated as vulnerability variables.

When an event occurs in a system or is bound to happen in the short term, evacuation measures must be applied and in some cases have to be designed in real time. Models and algorithms specified and calibrated in ordinary conditions $[6,7,8,9]$ cannot be directly applied in emergency conditions.

The emergency plan, in general, is defined considering two elements: each person knows exactly what to do (information); each person follows instructions exactly (coordination and organization). The assumptions are that the emergency plan is well coordinated and organized and the information is optimal. But who guarantees that: The available choice set for the authorities and operational forces is good, where the choice set is defined in terms of information, coordination and organization? The choice made is the best? The assumptions are true?

In real conditions very often to verify the quality of an evacuation strategy a real simulation on the real system is tested. This approach is very close to real conditions but is costly in terms of money, organization and people involved. During real experimentation if some of the scenario configurations have to be modified, a new real evacuation has to be organized. In real conditions also some new users could be present in the system and a priori experimentation is impossible.

Transportation risk analysis consists in developing a quantitative estimate of risk based on engineering assessments and possible mathematical techniques for combining estimates of incidents, consequences and frequencies [10]. In this paper, transportation risk analysis is proposed through a simulation approach with quantitative models where real conditions are reproducible on a computer. Computer simulation is cheaper than real simulation in terms of cost and time, and different strategies and scenarios can be tested. Real simulation may be useful to calibrate some parameters. For new users some hypotheses can be made; stochastic models may help in this direction.

This paper is developed with the following main objectives: (a) to formalize the risk problem with clear diversification in the definition of the vulnerability and exposure in a transportation system; thus the paper gives improvements in consolidated quantitative risk analysis models, especially transportation risk analysis models; (b) to specify a system of models for evacuation simulation and design.

In relation to the proposed objectives in this paper: (a) a general framework is reported with specific methods and models (section 2) for analyzing urban 
transportation system performances in emergency conditions when exogenous phenomena occur and for the specification of the risk function; (b) the evacuation problem could be studied in the standard simulation context of a "what if" approach or in the more recent optimization "what to" approach (section 3).

Some conclusions and indications for research developments are reported in section 4 .

\section{Risk function}

Risk can be defined as a cardinal measure of potential economic loss, human injury, or environmental damage in terms of both incident probability and the magnitude of the loss, injury, or damage [10].

Risk has to be assessed in all its components (probability, vulnerability and exposure) and a numeric value has to be obtained. This value can be considered a cardinal measure of the safety and security level. An increase in the risk level is equivalent to a reduction in safety and security. A general formulation for risk assessment in all its components has to be developed. Two types of risks can be defined: individual risk and societal risk. Individual risk is associated with a particular person or at a particular location; societal risk is associated with an activity to a particular population. In [10] risk assessment is proposed by means of quantitative analysis defining:

- for individual risk

A) the individual risk level that gives the risk level for a person at a particular location;

B) the average individual risk that gives the average risk to all the population;

C) the maximum individual risk that illustrates the highest risk to any one individual of a population;

- for societal risk

D) the societal risk level that gives the total risk level associated with an activity to a particular population.

Moreover, in [10] risk assessment is proposed by means of geographic (map) analysis:

- for individual risk

E) the individual risk contour that illustrates the geographical distribution of individual risk;

- for societal risk

F) the societal risk curve that illustrates the curve of the probability and the consequences of the total risk associated with an activity to a particular population.

Risk above a certain level (in one or more indicators) is considered intolerable and not justified and some measures have to be introduced to prevent the risk exceeding pre-defined risk level, named intolerable risk. The risk limit is not defined by scientific calculation but by observation of what society at present tolerates [11]. The change in intolerable risk during the years combined with recent events shows how the risk limit for society changes in time. 
Individual intolerable risk is defined as a maximum level that the individual accepts for the individual risk level indexes. Over these values there is an alarm level and below these values an acceptable level. The definition of individual intolerable risk can be extended to societal intolerable risk but there are some conceptual difficulties in defining this limit. This is due to the complexity of societal intolerable risk as there is difficulty allowing different types of events with the same level.

Starting from the definition of risk by which it depends on the probability (or the frequency) that the event occurs and on the magnitude of the loss, injury, or damage, in a simplified version societal risk $\mathrm{R}$ can be defined as:

$$
\mathrm{R}=\mathrm{P} \mathrm{M}
$$

where

- $\quad \mathrm{P}$ is the probability that an emergency event occurs;

- $\quad \mathrm{M}$ is the magnitude defined as a cardinal measure of the consequences for a particular population.

Eq. (1) can be also written in the form:

$$
\mathrm{R}=\mathrm{P} \mathrm{V} \mathrm{N}
$$

where

- $\mathrm{M}$ is defined as $\mathrm{M}=\mathrm{V} \mathrm{N}$

- $\mathrm{V}$ is vulnerability;

- $\mathrm{N}$ is exposure.

In the literature there is no clear distinction between vulnerability and exposure, especially in transportation systems. Hence in this paper we state the definition straightaway and propose a mathematical formulation.

The vulnerability of the system can be defined as the resistance of the infrastructures (material and immaterial) when the emergency occurs. Vulnerability is thus related only to the resistance of the infrastructures when the emergency occurs and there is no dependence on demand. Vulnerability is a supply characteristic. Examples of vulnerability are: the degree of resistance of a bridge when an emergency occurs; the period of time during which a data network and servers function properly before a crash. Vulnerability could also be connected to buildings that collapse on road surfaces or to traffic lights at junctions that are out of order due to the event.

The exposure of the system can be defined as the equivalent homogeneous weighted value of people, goods and infrastructures affected during and after the event. Exposure is a demand and demand/supply interaction characteristic. An example of exposure is the number of users in the system area that could die if not evacuated when an emergency occurs. To use the same example as above, exposure is also the value of the bridge and/or the buildings that collapse on the road surface and also the number of people on the bridge or inside the buildings and again that can be evacuated.

Considering eq. (1) two types of measure for risk reduction may be defined (Fig. 1): 
- $\quad$ prevention, which consists in reducing the level of $\mathrm{P}$;

- $\quad$ rotection, which consists in reducing the level of $\mathrm{M}$.

Currently, the reduction of $\mathrm{P}$ is possible only for some kinds of events which occur in relation to human activities (power failure, radiation leak, hazardous freight, etc.) and is the main objective of safety planners.

The magnitude $(\mathrm{M}=\mathrm{VN})$ can be reduced with two classes of measure (Fig. 1):

- $\quad$ resistance, which consists in reducing the level of $\mathrm{V}$;

- evacuation, which consists in reducing the level of $\mathrm{N}$.
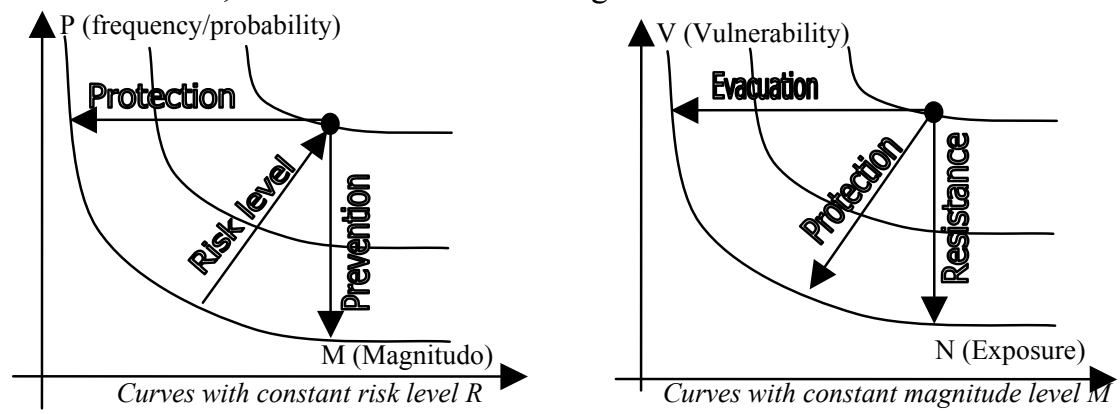

Figure 1: Possible measures for societal risk reduction.

In this paper a generalized formulation is proposed for societal risk assessment that goes beyond the traditional formulation where vulnerability and exposure are considered constant throughout the area studied. Having defined an emergency event $\mathrm{E}$ (for example a radiation leakage in a nuclear power station) in an area $\mathrm{T}$ (land around the power station), event $\mathrm{E}$ may occur in $\mathrm{T}$ with an intensity level in the range $\mathrm{L}_{\mathrm{E}}$ (for example the intensity of radiation emissions between two prefixed values), in the time slice $\Delta$ (for example in the next 2 years).

For each event a probability density function $p(\mathrm{x}, \mathrm{y}, \mathrm{z})$ can be defined where $\mathrm{x}$, $y$ and $z$ are respectively the variables for the intensity level in the range $L_{E}$, the area $\mathrm{T}$ and the time slice $\Delta$. The function $p$ is a probability function and can be defined as:

$$
\int_{\mathrm{x} \in \mathrm{L}_{\mathrm{E}}} \int_{\mathrm{y} \in \mathrm{T}} \int_{\mathrm{z} \in \Delta} p(\mathrm{x}, \mathrm{y}, \mathrm{z}) \mathrm{dz} d \mathrm{y} d \mathrm{x}=\mathrm{P}
$$

with the constraint $\mathrm{P} \in[0,1] . \mathrm{P}$ is the probability level that at least an event $\mathrm{E}$ may occur in $\mathrm{T}$, with an intensity level in the range $\mathrm{L}_{\mathrm{E}}$, in the time slice $\Delta$.

The function $\mathrm{p}$ is a probability density function and the value $\mathrm{p}(\mathrm{x}, \mathrm{y}, \mathrm{z}) \mathrm{dz} d \mathrm{~d}$ $\mathrm{dx}$ is the probability that the event $\mathrm{E}$ happens with intensity level between $\mathrm{x}$ and $\mathrm{x}+\mathrm{dx}$ in the surface area dy around point $\mathrm{y}$ in the time slice between $\mathrm{z}$ and $\mathrm{z}+$ $\mathrm{dz}$.

If the probability function $p$ assumes a value $\mathrm{p}$ in $\mathrm{L}_{\mathrm{E}}, \mathrm{T}$ and $\Delta$, the relation between $\mathrm{p}$ and $\mathrm{P}$ is: 


$$
\mathrm{p}\left(\int_{\mathrm{x} \in \mathrm{L}_{\mathrm{E}}} \mathrm{dx}\right)\left(\int_{\mathrm{y} \in \mathrm{T}} \mathrm{dy}\right)\left(\int_{\mathrm{z} \in \Delta} \mathrm{dz}\right)=\mathrm{P}
$$

The function can be obtained from the frequency that the event occurs if there are the available data. In this case different probability density functions can be tested with the Kolmogorov-Smirnov test in order to find the best distribution.

The level of magnitude that produces an event $\mathrm{E}$ with intensity level $\mathrm{x}$ at point $\mathrm{y}$ of $\mathrm{T}$ is $M(\mathrm{x}, \mathrm{y})$ given by the product of:

$$
M(\mathrm{x}, \mathrm{y})=V(\mathrm{x}, \mathrm{y}) N(\mathrm{x}, \mathrm{y})
$$

where

- vulnerability $V(\mathrm{x}, \mathrm{y})$ is the capacity of infrastructures (building, road, bridge, ....) at point $y$ of $T$ not to withstand event $E$ with intensity level $x$;

- exposure $N(\mathrm{x}, \mathrm{y})$ is the equivalent homogeneous weighted value of people, goods and infrastructures at point $\mathrm{y}$ of $\mathrm{T}$ affected during and after the event occurring with intensity level $\mathrm{x}$ in the range $\mathrm{L}_{\mathrm{E}}$.

With the given definitions and notations, assessments can be made with a set of indexes:

- for individual risk

A) the individual risk level, $R_{\mathrm{L}_{\mathrm{E}}, \mathrm{T}, \Delta}^{\mathrm{i}}(\mathrm{y})$, for event $\mathrm{E}$, at point $\mathrm{y}$ of area $\mathrm{T}$, with intensity level in the range $\mathrm{L}_{\mathrm{E}}$, in a time slice $\Delta$, can be defined as:

$$
R_{\mathrm{L}, \mathrm{T}, \Delta}^{\mathrm{i}}(\mathrm{y})=\int_{\mathrm{x} \in \mathrm{L}_{\mathrm{E}}} \int_{\mathrm{z} \in \Delta} M(\mathrm{x}, \mathrm{y}) p(\mathrm{x}, \mathrm{y}, \mathrm{z}) \mathrm{dz} d \mathrm{x}
$$

B) the average individual risk level, $R_{\mathrm{L}_{\mathrm{E}}, \mathrm{T}, \Delta}^{\mathrm{a}}(\mathrm{y})$, for event $\mathrm{E}$, in area $\mathrm{T}$, with intensity level in the range $\mathrm{L}_{\mathrm{E}}$, in a time slice $\Delta$, can be defined as:

$$
\mathrm{R}_{\mathrm{L}_{\mathrm{E}}, \mathrm{T}, \Delta}^{\mathrm{a}}=\int_{\mathrm{x} \in \mathrm{L}_{\mathrm{E}}} \int_{\mathrm{y} \in \mathrm{T}} \int_{\mathrm{z} \in \Delta} M(\mathrm{x}, \mathrm{y}) p(\mathrm{x}, \mathrm{y}, \mathrm{z}) \mathrm{dz} d \mathrm{y} d \mathrm{x} /\left(\int_{\mathrm{y} \in \mathrm{T}} \mathrm{dy}\right)
$$

C) the maximum individual risk level $R_{\mathrm{L}_{\mathrm{E}}, \mathrm{T}, \Delta}^{\mathrm{m}}(\mathrm{y})$, for event $\mathrm{E}$, in area $\mathrm{T}$, with intensity level in the range $\mathrm{L}_{\mathrm{E}}$, in a time slice $\Delta$, can be defined as:

$$
\mathrm{R}_{\mathrm{L}_{\mathrm{E}}, \mathrm{T}, \Delta}^{\mathrm{m}}=\max _{\mathrm{y} \in \mathrm{T}} R_{\mathrm{L}_{\mathrm{E}}, \mathrm{T}, \Delta}^{\mathrm{i}}(\mathrm{y})=\max _{\mathrm{y} \in \mathrm{T}}\left(\int_{\mathrm{x} \in \mathrm{L}_{\mathrm{E}}} \int_{\mathrm{z} \in \Delta} M(\mathrm{x}, \mathrm{y}) p(\mathrm{x}, \mathrm{y}, \mathrm{z}) \mathrm{dz} \mathrm{dx}\right)
$$

- for societal risk

D) the general cardinal measure of societal risk for event $E$ in $T$, with intensity level in the range $\mathrm{L}_{\mathrm{E}}$, in a time slice $\Delta$, can be defined as:

$$
\mathrm{R}_{\mathrm{L}_{\mathrm{E}}, \mathrm{T}, \Delta}=\int_{\mathrm{y} \in \mathrm{T}} R_{L_{E}, T, \Delta}^{i}(y) \mathrm{dy}=\int_{\mathrm{x} \in \mathrm{L}_{\mathrm{E}}} \int_{\mathrm{y} \in \mathrm{T}} \int_{\mathrm{z} \in \Delta} M(\mathrm{x}, \mathrm{y}) p(\mathrm{x}, \mathrm{y}, \mathrm{z}) \mathrm{dz} d \mathrm{y} d \mathrm{x}
$$

Considering eq. (5) societal risk can be expressed as:

$$
\mathrm{R}_{\mathrm{L}_{\mathrm{E}}, \mathrm{T}, \Delta}=\int_{\mathrm{x} \in \mathrm{L}_{\mathrm{E}}} \int_{\mathrm{y} \in \mathrm{T}} \int_{\mathrm{z} \in \Delta} V(\mathrm{x}, \mathrm{y}) N(\mathrm{x}, \mathrm{y}) p(\mathrm{x}, \mathrm{y}, \mathrm{z}) \mathrm{dz} d \mathrm{y} d \mathrm{x}
$$

Eq. (2), considered for risk assessment, is a particular case of eq. (6) under several restrictive hypotheses.

In the hypothesis of

- constant vulnerability $V(\mathrm{x}, \mathrm{y})$ with respect to $\mathrm{T}$ and in the range $\mathrm{L}_{\mathrm{E}}$ and equal to $\mathrm{V}$;

- constant exposure $N(\mathrm{x}, \mathrm{y})$ in $\mathrm{T}$ and in the range $\mathrm{L}_{\mathrm{E}}$ and equal to $\mathrm{N}$; 
societal risk assumes the simple form:

$$
\mathrm{R}_{\mathrm{L}_{\mathrm{E}}, \mathrm{T}, \Delta}=\mathrm{V} \mathrm{N} \int_{\mathrm{x} \in \mathrm{L}_{\mathrm{E}}} \int_{\mathrm{y} \in \mathrm{T}} \int_{\mathrm{z} \in \Delta} p(\mathrm{x}, \mathrm{y}, \mathrm{z}) \mathrm{dz} d \mathrm{ydx}
$$

Considering eq. (3), the risk reported in (7) is equal to:

$$
\mathrm{R}_{\mathrm{L}_{\mathrm{E}}, \mathrm{T}, \Delta}=\mathrm{P} \text { V N }
$$

Eq. (8) is equal to eq. (2) but they are equivalent only in the particular hypothesis described.

\section{Risk exposure in a transportation system}

To be consistent with the main objectives defined, in this paper we consider that the risk has to be evaluated and reduced when an event happens or is bound to happen in the short term and an evacuation plan has to be generated (also in real time). Hence the risk is formulated taking the following into account:

- an event happens with a predefined intensity level and in a homogeneous area with respect to the event;

- vulnerability $\mathrm{V}(\mathrm{x}, \mathrm{y})$ is constant.

An analysis of the effect of frequency or probability and vulnerability on the risk function may be found in papers belonging to other research areas. For frequency or probability, examples of events and theories are: for earthquakes, geotechnical and geologic theory; for chemical effects, chemistry theory; for terrorist attacks, policing and security theories. For vulnerability some examples of structures and theories are: for building vulnerability, construction theory; for vehicles, mechanical theory.

Exposure in the past was not considered a problem, nor was evacuation studied with a quantitative approach. Nowadays, exposure reduction with evacuation design has to be developed in transportation science.

Starting from the position given, a general formulation for designing transportation systems can be developed. The aim is to minimize the risk function $R$ as it is defined in eq. (6). It is supposed that for each event $\mathrm{E}$, with intensity level in the range $\mathrm{L}_{\mathrm{E}}$, in an area $\mathrm{T}$ :

- the vulnerability function $V$ cannot be modified and is given equal to $\mathrm{V}$ (constant values in $\mathrm{L}_{\mathrm{E}}$ and $\mathrm{T}$ );

- the exposure function $N$ is constant with respect to $\mathrm{x}$ in the range $\mathrm{L}_{\mathrm{E}}$ and equal to the function $N^{*}(\mathrm{y})$;

- the probability function $p$ cannot be modified and is given and equal to the constant value $\mathrm{p}$ in $\mathrm{L}_{\mathrm{E}}$, $\mathrm{T}$ and $\Delta$.

Considering these hypotheses, societal risk $R$ reported in eq. (6), given eq. (4) is:

$$
\mathrm{R}_{\mathrm{L}_{\mathrm{E}}, \mathrm{T}, \Delta}=\mathrm{Vp}\left(\int_{\mathrm{x} \in \mathrm{L}_{\mathrm{E}}} \mathrm{dx}\right)\left(\int_{\mathrm{z} \in \Delta} \mathrm{dz}\right) \int_{\mathrm{y} \in \mathrm{T}} N^{*}(\mathrm{y}) \mathrm{dy}=\mathrm{V} \mathrm{P}\left(\int_{\mathrm{y} \in \mathrm{T}} N^{*}(\mathrm{y}) \mathrm{dy}\right) /\left(\int_{\mathrm{y} \in \mathrm{T}} \mathrm{dy}\right)
$$

with the hypothesis that the function $N^{*}(\mathrm{y})$ allows the triple integral to separated into three single integral.

For $N^{*}(\mathrm{y})$, in relation to the optimization problem considered, different specific exposure functions can be defined as objective functions to identify 
evacuation measures in transportation systems. The area system and the transportation supply relative to area $\mathrm{T}$ can be discretized in a finite number of homogeneous areas with respect to exposure represented by:

- links representing sections of road and relative area activities and buildings giving onto them (if a road is not homogeneous in terms of exposure the sections are represented with different links in series);

- nodes representing the junctions between links, the fictitious points (r) where the origin of the user journey towards the assembly centres is assumed to be concentrated, the assembly centres (s) (safe points).

It is thus possible to represent transportation supply by a graph consisting of an ordinate pair of sets, a set of elements called nodes ( $i$ or $j$ ) and a set of pairs of nodes $(i, j)$ called arcs or links that are homogeneous with respect to the evacuation. To each link may be associated:

- a link flow $f_{i j}$ (f being the link flow vector) which represents the average number of users using the link in the time unit; the flow vector can only assume values belonging to its feasibility set denoted by $\mathrm{S}_{\mathrm{f}}$;

- a link cost scalar function $c_{\mathrm{ij}}$ (f) (c being the link cost vector) which allows us to calculate the average transport cost of each link corresponding to a link flow vector.

We may associate to each loop-less path $\mathrm{k}$ of the graph connecting the fictitious points to the assembly centres:

- a path flow $h_{k}$ (h being the flow path vector) which represents the average number of users who, in the time unit, use path $\mathrm{k}$ from their origin (in a fictitious point) to their destination (in an assembly center);

- a path cost $g_{k}$ (g being the cost path vector) which represents the average cost of users who, in the time unit, use path $\mathrm{k}$ from their origin (in a fictitious point) to their destination (in an assembly center).

The path cost $\mathrm{g}_{\mathrm{k}}$ is the sum of the link $\operatorname{cost} c_{\mathrm{ij}}(\mathbf{f})$ that belong $(\in)$ to path $\mathrm{k}: g_{k}$ $=\sum_{i j \in k} \mathrm{c}_{i j}(\boldsymbol{f})$. Considering the binary index $\delta_{\mathrm{ij}, \mathrm{k}}$ that is 1 if link ij belongs to path $\mathrm{k}$ and 0 otherwise, the path cost $\mathrm{g}_{\mathrm{k}}$ can be also written as the sum of the $\operatorname{cost} c_{\mathrm{ij}}(\mathbf{f})$ of links ij that belong to path $\mathrm{k}$ :

$$
\mathrm{g}_{\mathrm{k}}=\Sigma_{\mathrm{ij}} \delta_{\mathrm{ij}, \mathrm{k}} c_{\mathrm{ij}}(\mathbf{f})
$$

The link flow $\mathrm{f}_{\mathrm{ij}}$ is the sum of the path flow gk that contains $(\supset)$ the link $\mathrm{ij}: f_{i j}=$ $\Sigma_{k \rightarrow i j} h_{k}$. Considering the binary index $\delta_{\mathrm{ij}, \mathrm{k}}$ the link flow $\mathrm{f}_{\mathrm{ij}}$ can be also written as the sum of the flow $h_{k}$ of paths $h$ that contain the link ij:

$$
\mathrm{f}_{\mathrm{ij}}=\Sigma_{\mathrm{k}} \delta_{\mathrm{ij}, \mathrm{k}} \mathrm{h}_{\mathrm{k}}
$$

In this context the integral reported in eq. (9) to assess the risk can be transformed in a sum relative to the homogeneous elements (nodes and links). The exposure $N^{*}(\mathrm{y})$ in T can be evaluated separately for each homogeneous zone $r$ as the sum of the cost for all users to reach the safe points $s$, departing from $r$ and considering all the paths $(\Rightarrow)$ that connect $r$ and $s$ :

$$
N^{*}(\mathrm{r})=\Sigma_{\mathrm{s}} \sum_{\mathrm{k} \Rightarrow \mathrm{rs}} \mathrm{g}_{\mathrm{k}} \mathrm{h}_{\mathrm{k}}
$$


Global exposure evaluated in $\mathrm{T}$ reported in eq. (9) $\left(\int_{\mathrm{y} \in \mathrm{T}} N^{*}(\mathrm{y})\right.$ dy) can be expressed as the sum of $N^{*}(\mathrm{r})$ in all the homogeneous zones $\mathrm{r}$ :

$$
\int_{\mathrm{y} \in \mathrm{T}} N^{*}(\mathrm{y}) \mathrm{dy}=\Sigma_{\mathrm{r}} \mathrm{N}^{*}(r)=\Sigma_{\mathrm{r}} \Sigma_{\mathrm{s}} \Sigma_{\mathrm{k} \Rightarrow \mathrm{rs}} \mathrm{g}_{\mathrm{k}} \mathrm{h}_{\mathrm{k}}
$$

Considering the paths $\mathrm{k}$ that connect all the origins $\mathrm{r}$ to all the destinations $\mathrm{s}$ is equivalent to considering all the path $\mathrm{k}$. The previous equation can be written as:

$$
\int_{\mathrm{y} \in \mathrm{T}} N^{*}(\mathrm{y}) \mathrm{dy}=\Sigma_{\mathrm{k}} \mathrm{g}_{\mathrm{k}} \mathrm{h}_{\mathrm{k}}
$$

Considering the relation between path costs and link costs, eq. (10), and path flows and link flows, eq. (11), global exposure can also be written as:

$$
\int_{\mathrm{y} \in \mathrm{T}} N^{*}(\mathrm{y}) \mathrm{dy}=\Sigma_{\mathrm{ij}} c_{\mathrm{ij}}(\mathbf{f}) \mathrm{f}_{\mathrm{ij}}=\mathbf{c}^{\mathrm{T}} \mathbf{f}
$$

Societal risk reported in eq. (9) finally is:

$$
\mathrm{R}_{\mathrm{L}_{\mathrm{E}}, \mathrm{T}, \Delta}=\mathrm{V} P\left(\mathbf{c}^{\mathrm{T}} \mathbf{f}\right) /\left(\int_{\mathrm{y} \in \mathrm{T}} \mathrm{dy}\right)
$$

Other risk exposure can be considered, such as evacuation time or the time at which the last vehicle exits from the network.

In a transportation system and with the previous hypotheses (constant values of the function $V, p$ and the function $N$ constant in $\mathrm{L}_{\mathrm{E}}$ ), the minimization of the risk function $R$ is expressed as the minimization of the exposure function $N^{*}(\mathrm{y})$ with the integral throughout area $\mathrm{T}$. The optimization problem consists in obtaining the flows $\mathbf{f}^{*}$ ( $\arg \min$ ) that minimize the risk function (13):

$$
\mathbf{f}^{*}=\arg \min _{\mathbf{f} \in \mathrm{S}_{\mathrm{f}}} \mathrm{R}_{\mathrm{L}_{\mathrm{E}}, \mathrm{T}, \Delta}=\arg \min _{\mathbf{f} \in \mathrm{Sf}_{\mathrm{f}}} \mathrm{V} \mathrm{P}\left(\mathbf{c}^{\mathrm{T}} \mathbf{f}\right)\left(\int_{\mathrm{y} \in \mathrm{T}} \mathrm{dy}\right)
$$

From a transport point of view, the terms $\mathrm{V}$ and $\mathrm{P}$ are supposed constant as other research areas study the problem to reduce them. The $\int_{\mathrm{y} \in \mathrm{T}}$ dy is the size of $\mathrm{T}$ and is constant. The optimization problem (14) is equivalent to the following:

$$
\mathbf{f}^{*}=\arg \min _{\mathbf{f} \in \mathrm{S}_{\mathrm{f}}} \mathrm{R}_{\mathrm{L}_{\mathrm{E}}, \mathrm{T}, \Delta}=\arg \min _{\mathbf{f} \in \mathrm{S}_{\mathrm{f}}} \mathbf{c}^{\mathrm{T}} \mathbf{f}
$$

Risk $\mathrm{R}_{\mathrm{L}_{\mathrm{E}}, \mathrm{T}, \Delta}$ can be valued considering the optimal flow $\mathrm{f}^{*}$ in eq. (13).

The problem reported in eq. (15) is a formulation in terms of design (or "what to") approach where an objective function (in this context $\mathbf{c}^{\mathrm{T}} \mathbf{f}$ represents the sum of the cost for all users to reach the safe points) is minimised. At the minimum for the objective function, the path and link flows and costs are obtained. The flow and the costs are carried out with an optimum set of path choice by the users. This optimal path must be followed by users minimize global exposure.

The conditions for the existence and uniqueness of the solution for the eq. (15) are relative to the cost function that has to be continuous, differentiable and monotone and are demonstrated in [9].

In the problem, vulnerability may not be constant. The problem can be solved with different levels of vulnerability and different optimal solutions can be obtained. This is possible for example if the risk and the optimal system configuration have to be defined in real time after an event happens on the system. In this context the infrastructural configuration is defined in real time and the new optimal configuration can be generated. 


\section{Conclusions}

In this paper a general model for risk evaluation in transportation systems is proposed and vulnerability and exposure are explained. Methods for evacuation are proposed for designing evacuation in a road urban network system in emergency conditions. Research for the analysis and modelling of transportation systems in emergency conditions requires further studies. In emergency conditions there is the need to develop new methods and rearrange standard procedures such as: network vulnerability analysis in terms of the "safety coefficient" of the supply system in relation to events with different levels of danger and different probabilities of fulfilment; specification and calibration of link cost functions to use in system simulation in over-saturation conditions in the periods analysed; specification and calibration of demand models for the different choice levels for users and for the public decision-maker. In this paper it is evident that the definition of the best scenario emerges only from the simulation of pre-defined scenarios, taking into account supply, demand and their interaction. The management of emergency conditions and evacuation of an urban area must necessarily be supported by quantitative analyses.

\section{References}

[1] R. Goldblatt, R., "Development of Evacuation Time Estimates for the Davis Nuclear Power Station", (Tech. Rep. 329 KLD, 1993).

[2] F. Russo, Vitetta A., "The road network design problem to improve the safety during exogenous flow perturbations", Proceedings of the $29^{\text {th }}$ ISATA Conference (Florence, 1996).

[3] R. Goldblat, "Evacuation planning. Human factor and traffic engineering perspectives". Proceedings of the European Transport Conference (Strasbourg, 2004).

[4] University of Maryland, Fire and Rescue Institute - http://www.mfri.org.

[5] M. Di Gangi, A. Luongo, R. Polidoro, "Una procedura di carico dinamico per la valutazione dei piani di evacuazione", Proceedings of $2^{\circ}$ seminario scientifico su Metodi e Tecnologie dell'Ingegneria dei Trasporti, Università di Reggio Calabria, (Franco Angeli, Milan, 2000).

[6] M. Ben Akiva, S. Lerman, Discrete choice analysis: theory and application to travel demand (MIT Press, Cambridge, MA, 1985).

[7] Y. Sheffi, Urban transportation networks (Prentice Hall, Englewood Cliff, NJ, 1985).

[8] K. Train K., Discrete choice methods with simulation (Mit Press Cambridge, Massachusetts, 2003).

[9] E. Cascetta, Transportation systems engineering: theory and methods (Kluwer, Academic Press, 2001).

[10] CCPS, Guidelines for chemical transportation risk analysis (American Institute of Chemical Engineers, New York, 1995).

[11] Health and Safety Commission, Major hazard aspects of the transport of dangerous substances. Report and appendices (HMSO, London, 1991). 